\title{
Modeling Efficient XOR-Based Hash Functions for Cache Memories`
}

\author{
Sung-Jin Cho ${ }^{1}$, Un-Sook Choi ${ }^{2}$, Yoon-Hee Hwang ${ }^{3}$, and Han-Doo Kim ${ }^{4}$ \\ ${ }^{1}$ Division of Mathematical Sciences, \\ Pukyong National University, Busan 608-737, Korea \\ sjcho@pknu.ac.kr \\ ${ }^{2}$ Department of Multimedia Engineering, Tongmyoung University \\ Busan 608-711, Korea \\ choies@pknu .ac . kr \\ ${ }^{3}$ Department of Information Security, Graduate School, \\ Pukyong National University, Busan 608-737, Korea \\ yhhwang@pknu.ac.kr \\ ${ }^{4}$ School of Computer Aided Science, Inje University \\ Gimhae 621-749, Korea \\ mathkhd@inje.ac.kr
}

\begin{abstract}
In this paper, we design new XOR-based hash functions, which compute each set index bit as XOR of a subset of the bits in the address. These are conflict-free hash functions which are different types according to $m$ is even or odd.
\end{abstract}

\section{Introduction}

Hash functions are used in processors to augment the bandwidth of an interleaved multibank memory or to enhance the utilization of a prediction table or a cache 1. Bank conflicts can severely reduce the bandwidth of an interleaved multibank memory and conflict misses increase the miss rate of a cache. Therefore it is important that a hash function has to succeed in spreading the most frequently occurring patterns over all indices. Vandierendonck et al. 2] constructed XOR-based hash functions which provided conflict-free mapping for a number of patterns for multibank memories and skewed-associative caches. Their functions map $2 m$ bits to $m(=2 k)$ bits which are conflict free. But they didn't construct hash functions which are conflict free when $m$ is odd. Also they constructed two XOR-based hash functions for skewed-associative caches. But the degree of interbank dispersion between two hash functions is less than $2 m$, which is the maximum degree between them. So they changed the basis of one hash function to overcome this problem.

In this paper, we design new XOR-based hash functions, which compute each set index bit as XOR of a subset of the bits in the address by using the concepts of rank and null space ([3], 4] ). These are conflict-free hash functions which are

\footnotetext{
^ This work was supported by KOSEF:R01-2006-000-10260-0.
} 
different types according to $m$ is even or odd. To apply the constructed hash functions to the skewed-associative cache, we show that the degree of interbank dispersion between two hash functions is maximal.

\section{Modeling Efficient XOR-Based Hash Functions}

A hash function is a function from $\left\{0, \cdots, 2^{n}-1\right\}(:=\mathbf{A})$ of $n$-bit addresses to $\left\{0, \cdots, 2^{m}-1\right\}(:=\mathbf{S})$ of $m$-bit indices $(m<n)$. An $n$-bit address a is represented by a bit vector $a_{1}, \cdots, a_{n}$. A hash function mapping $n$ bits to $m$ bits is represented as a binary matrix $H$ with $n$ rows and $m$ columns. Since $H$ is surjective, the image of $\mathbf{A}$ is $\mathbf{S}$. Therefore $\operatorname{rank}(H)=\operatorname{dim}(\mathbf{S})=m$, where $\operatorname{dim}(\mathbf{S})$ is the dimension of $\mathbf{S}$. The bit on the $i$-th row and the $j$-th column is 1 when address bit $a_{i}$ is an input to the XOR-gate computing the $j$-th set index bit. Consequently, the computation of the set index $\mathbf{s}$ can be expressed as the vector-matrix multiplication over $G F(2)$, where addition is computed as XOR and multiplication is computed as logical AND, denoted by $\mathbf{s}=\mathbf{a} H$.

Since matrices are considered as linear transformations, they can be characterized by their null spaces. The null space of a matrix $H$ is the set of all addresses which map to index $\mathbf{0}$, namely $N(H)=\{\mathbf{x}: \mathbf{x} H=\mathbf{0}\}$.

In this section we model efficient XOR-based hash functions when the number of address bits is $2 m$, where $m$ is even or odd. We propose the new model of XOR-based hash functions for two cases. We give XOR-based hash functions by the following.

Definition 2.1. We define XOR-based hash functions of four types as the following:

(i) $\quad m=2 k-1 \quad(k \in \mathbf{N})$, where $\mathbf{N}$ is the set of all positive integers. $H_{1}=\left(T_{1}, I_{2 k-1}\right)^{t}, H_{3}=\left(T_{3}, I_{2 k-1}\right)^{t}$, where $I_{2 k-1}$ is the identity matrix,

$T_{1}=\left(t_{i j}\right)_{(2 k-1) \times(2 k-1)}, \quad t_{i j}= \begin{cases}1, & \text { if } 1 \leq i \leq k, 1 \leq j \leq k-i+1, \\ 1, & \text { if } 1 \leq i \leq 2 k-1, j=2 k-i, \\ 0, & \text { otherwise }\end{cases}$ $T_{3}=\left(t_{i j}\right)_{(2 k-1) \times(2 k-1)}, \quad t_{i j}= \begin{cases}1, & \text { if } i=j=1 \\ 1, & \text { if } 1 \leq i \leq 2 k-1, j=2 k-i, \\ 1, & \text { if } k \leq i \leq 2 k-1,3 k-i-1 \leq j \leq 2 k-1, \\ 0, & \text { otherwise }\end{cases}$ (ii) $\quad m=2 k \quad(k \in \mathbf{N})$

$H_{2}=\left(T_{2}, I_{2 k}\right)^{t}, H_{4}=\left(T_{4}, I_{2 k}\right)^{t}$, where $I_{2 k}$ is the identity matrix, $T_{2}=\left(t_{i j}\right)_{(2 k) \times(2 k)}, \quad t_{i j}= \begin{cases}1, & \text { if } 1 \leq i \leq k, i \leq j \leq k, \\ 1, & \text { if } 1 \leq i \leq 2 k-1, j=2 k-i, \\ 0, & \text { otherwise }\end{cases}$

$T_{4}=\left(t_{i j}\right)_{(2 k) \times(2 k)}, \quad t_{i j}= \begin{cases}1, & \text { if } k+1 \leq i \leq 2 k, k+1 \leq j \leq i, \\ 1, & \text { if } 1 \leq i \leq 2 k-1, j=2 k-i, \\ 0, & \text { otherwise }\end{cases}$ 
Theorem 2.2. (i) All $m \times m$ matrices $T_{i}^{\prime} s$ in Definition 2.1 are nonsingular, where $i=1,2,3,4$.

(ii) Each $T_{i} \oplus I$ is nonsingular, where $T_{i}$ is in Definition 2.1.

Theorem 2.3. Let $H=\left(T, I_{m}\right)^{t}$ be the hash function in Definition 2.1, where $T=\left(\mathbf{t}_{\mathbf{1}}, \mathbf{t}_{\mathbf{2}}, \cdots, \mathbf{t}_{\mathbf{m}}\right)^{t}$ and $I_{m}=\left(\mathbf{e}_{\mathbf{1}}, \mathbf{e}_{\mathbf{2}}, \cdots, \mathbf{e}_{\mathbf{m}}\right)^{t}$. And let

$$
M_{i j}=\left(\mathbf{t}_{\mathbf{m}+\mathbf{1}-\mathbf{i}}, \cdots, \mathbf{t}_{\mathbf{m}-\mathbf{1}}, \mathbf{t}_{\mathbf{m}}, \mathbf{e}_{\mathbf{m}+\mathbf{1}-\mathbf{j}}, \cdots, \mathbf{e}_{\mathbf{m}}\right)^{t}
$$

where $i+j=m$. Here $A^{t}$ is the transpose of $A$. Then $\operatorname{rank}\left(M_{i j}\right)=m$.

By Theorems 2.2 and 2.3, the modeled XOR-based hash functions such as the functions defined in Definition 2.1 map the patterns(rows, columns, (anti)diagonal, and rectangles) without conflicting.

\section{Conflict-Free Mapping in 2-Way Skewed-Associative Caches}

In this section, we construct XOR-based hash functions for a 2-way skewedassociative cache of the same size that maps the same patterns conflict-free.

Definition 3.1. We define the degree of interbank dispersion(DID) between two XOR-based hash functions $H_{1}, H_{3}$ by $2 m \times m$

$$
D I D\left(H_{1}, H_{3}\right)=\operatorname{rank}\left[H_{1} H_{3}\right]
$$

\begin{tabular}{|c|c|c|c|c|c|c|c|c|c|}
\hline & \multicolumn{8}{|c|}{ Value of $\mathrm{H}_{1}$} \\
\hline & & 0 & 1 & 2 & 3 & 4 & 5 & 6 & 7 \\
\hline \multirow{8}{*}{ 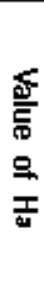 } & 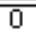 & $0: 0$ & $5: 2$ & $4: 5$ & 1:7 & $7: 1$ & $2: 3$ & $3: 4$ & $6: 6$ \\
\hline & 1 & & $0: 1$ & $1: 6$ & $4: 4$ & $2: 2$ & $7: 0$ & 6: 7 & $3: 5$ \\
\hline & & $4: 7$ & $1: 5$ & $0: 2$ & $5: 0$ & $3: 6$ & $6: 4$ & $7: 3$ & 2: \\
\hline & 3 & 4 & $4: 6$ & $5: 1$ & $0: 3$ & $6: 5$ & $3: 7$ & $2: 0$ & $7: 2$ \\
\hline & 4 & 5 & $2: 7$ & $3: 0$ & $6: 2$ & $0: 4$ & $5: 6$ & $4: 1$ & $1: 3$ \\
\hline & 5 & $2: 6$ & $7: 4$ & $6: 3$ & $3: 1$ & $5: 7$ & $0: 5$ & $1: 2$ & $4: 0$ \\
\hline & & $3: 2$ & $6: 0$ & $7: 7$ & $2: 5$ & $4: 3$ & $1: 1$ & $0: 6$ & $5: 4$ \\
\hline & 7 & $6: 1$ & $3: 3$ & $2: 4$ & $7: 6$ & $1: 0$ & $4: 2$ & $5: 5$ & $0: 7$ \\
\hline
\end{tabular}

Fig. 1. Illustration of $H_{1}$ and $H_{3}$ and interbank dispersion

The hash functions of a 2-way skewed-associative cache should be designed such that the DID is maximal for every pair of hash functions. Vandierendonck et al. 2 defined the DID between two hash functions $H_{1}$ and $H_{3}$ by using the concepts of supremum(infimum) of two functions and the concept of the dimension of column space. But we defined the DID by using only the concept of the rank of the augmented matrix $\left[H_{1} H_{3}\right]$. Every address in main memory is mapped to a set in bank 1 by $H_{1}$ and to a set in bank 2 by $H_{3}$. These functions 
are illustrated in a two-dimensional plot(Fig. 1). Each axis is labeled with the possible set indices for that bank. Every address is displayed in the grid in a position that corresponds to its set indices in each bank. For two vectors $x$ and $y$ in the same row, $x H_{3}=y H_{3}$ but $x H_{1} \neq y H_{1}$. Similarly, for two vectors $u$ and $v$ in the same column, $u H_{1}=v H_{1}$ but $u H_{3} \neq v H_{3}$. Therefore this skewedassociative cache can avoid conflict by $H_{1}$ and $H_{3}$. Fig. 1 shows that the DID of $H_{1}$ and $H_{3}$ is maximal. Also the element (5:3) represents (101 011).

The following theorem characterize the maximality of the DID of $H_{1}$ and $H_{3}$.

Theorem 3.3. The degree of interbank dispersion between $H_{1}$ and $H_{3}$ is maximal if and only if $N\left(\left[H_{1} H_{3}\right]\right)=\{\mathbf{0}\}$.

Lemma 3.4. Let $T_{1}$ and $T_{3}$ be matrices in Definition 3.1, where $m=2 k-1(k \geq 3)$. Then $\operatorname{rank}\left[T_{1} \oplus T_{3}\right]=m$.

The following theorem shows that the DID between the proposed hash functions $H_{1}$ and $H_{3}$ is maximal. We can see that these functions map $2 m$-bit address to $m$-bit set index without conflict for a 2 -way skewed-associative cache.

Theorem 3.5. Let $H_{1}=\left(T_{1}, I_{m}\right)^{t}$ and $H_{3}=\left(T_{3}, I_{m}\right)^{t}$ for $T_{1}$ and $T_{3}$ are matrices in Definition 2.1, where $m=2 k-1(k \geq 3)$. Then $\operatorname{rank}\left[H_{1} H_{3}\right]=2 m$.

Corollary 3.6. Let $H_{1}=\left(T_{1}, I_{m}\right)^{t}$ and $H_{3}=\left(T_{3}, I_{m}\right)^{t}$ for $T_{1}$ and $T_{3}$ are matrices in Definition 2.1, where $m=2 k-1(k \geq 3)$. Then $N\left(\left[\begin{array}{ll}H_{1} & H_{3}\end{array}\right]\right)=\{\mathbf{0}\}$.

We can show that Theorem 3.5 and Corollary 3.6 hold for $H_{2}$ and $H_{4}$, where $m=2 k$.

\section{Conclusion}

In this paper we designed new XOR-based hash functions by using the concepts of rank and null space. We constructed conflict-free hash functions which are different types according to $m$ is even or odd. We showed that the DID between the proposed hash functions is maximal. By the result we showed that these functions map without conflict for a 2-way skewed-associative cache.

\section{References}

1. A. Seznec, A New Case for Skewed-Associativity, Technical Report PI-1114, IRISH (1997)

2. Hans Vandierendonck and Koen De Bosschere, XOR-Based Hash Functions, IEEE Trans. Computers 54 (2005) 800-812

3. S.J. Cho, U.S. Choi, Y.H. Hwang, Y.S. Pyo, H.D. Kim and S.H. Heo, Computing Phase Shifts of Maximum-Length 90/150 Cellular Automata Sequences, Lecture Notes in Computer Science 3305 (2004) 31-39

4. S.J. Cho, U.S. Choi and H.D. Kim, Behavior of Complemented CA whose Complement Vector is Acyclic in a Linear TPMACA, Mathematical and Computer Modelling 36 (2002) 979-986 